\title{
Characterization of Precipitates with the Atom Probe
}

\author{
M.K. Miller and R.C. Thomson* \\ Microscopy and Microanalytical Sciences Group, Metals and Ceramics Division, Oak Ridge National \\ Laboratory, P.O. Box 2008, Oak Ridge, TN 37831-6376, U.S.A. \\ * Loughborough University, Loughborough, Leicestershire LE11 3TU, U.K.
}

\begin{abstract}
The parameters that can be measured from atom probe random area analyses of small spherical precipitates and platelets have been examined with a combination of simulation and geometrical models. The results indicate that the probe aperture should be less than $50 \%$ of the diameter of the precipitate in order to reliable measure the true composition in a significant proportion of precipiates.
\end{abstract}

\section{INTRODUCTION}

One of the advantages of the modern atom probe over other microanalytical tools is its ability to detect and analyze precipitates that are less than $10 \mathrm{~nm}$ in diameter. This type of characterization is normally achieved by performing a random area analysis through the specimen and collecting the atoms that originate in a cylinder of material. The raw atom-by-atom data is divided into small blocks of atoms from which the composition is calculated. The resulting composition profile is a function of both material parameters such as the precipitate size, morphology and number density, and instrumental parameters such as the effective diameter of the probe aperture, detection efficiency of the single atom detector, as well as the number of atoms used to define the block size, and the number of atoms collected. In cases in which analyses of non-spherical precipitates are performed, the orientation relationship between the cylinder of analysis and the precipitate is also an important parameter.

A series of analyses of a thin $\mathrm{NiBe}$ plate, demonstrates the problem under consideration. A pair of field ion micrographs of thin brightly-imaging $\mathrm{NiBe}$ platelets in the $\gamma^{\prime} \mathrm{Ni}_{3} \mathrm{Al}$ phase of a model superalloy containing 10 at. \% Be are shown in Fig. 1. A series of atom probe composition profiles across these two precipitates is shown in Fig. 2. On the thicker precipitate, Fig. 2a, a large probe aperture (where the effective radius of the probe aperture $r_{a p}$ is signficantly less than the thickness of the plate, $w$ ) may be used and the correct $\mathrm{NiBe}$ stoichiometry is obtained. If this large probe aperture is used on the smaller precipitate (where $r_{a p}$ is similar to w), Fig. $2 \mathrm{~b}$, the true concentration is not measured due to simultaneous sampling of the precipitate and matrix. If a smaller probe aperture is used (where $r_{a p}$ is less than w), Fig. $2 c$, the correct NiBe stoichiometry is obtained but the number of atoms collected decreases and the error in the measurement increases. Similar results are obtained in the case of spherical precipitates.

The effectiveness of the atom probe in the characterization of ultrafine precipitates has been investigated with a combination of the atom probe simulator and geometrical models. This investigation is limited to cases where the size of the precipitate and the effective size of the probe aperture are similar. Cases in which selected area analysis of the precipitate is possible are not considered in this paper since the composition of the precipitate is not affected by simultaneous sampling of the precipitate and the matrix. It should be noted that this approach may also be used in the analysis of data from 3-dimensional atom probes.

\section{ATOM PROBE SIMULATOR}

The atom probe simulator is a set of computer programs that permits any microstructure to be generated and visualized in a computer and sampled with a cylinder of analysis [1]. The atom probe simulator consists of two main components. The first component is used to construct a desired microstructure by defining the spatial coordinates and mass-to-charge ratio of all the atoms in a given volume. This is accomplished by a series of computer programs that each generate a specific type of microstnicture. This modular approach permits relatively simple modifications to be made to model other types of microstructures. For example, programs 
have been developed to model precipitation, ordered microstructures, and interfaces. The second component takes these data and visualizes and analyzes them on a graphics-oriented workstation. The visualization part of the system has been integrated into a commercial software package. The system can process a volume containing in excess of $10^{6}$ atoms. It should be noted that artefacts arising from the field evaporation process (such as local magnification effects) are not taken into account in this method.

The analysis of a monodispersion of ultrafine spherical precipitates is considered. The case of ultrafine spherical precipitates is of great technological importance in the nuclear industry due to the embrittlement that occurs in pressure vessel steels during service due to high copper contents. Therefore, the alloy system chosen for this investigation was a model of the early stages of copper precipitation in pressure vessel steels. The simulation variables included precipitate and matrix compositions, size and number density of the precipitates, and detection efficiency of the single atom detector. A simulation of copper precipitation in the $\mathrm{Fe}-\mathrm{Cu}$ system with randomly-distributed spherical non-impinging 2-nm-diameter coherent bec precipitates with a composition of $\mathrm{Fe}-90 \% \mathrm{Cu}$ in a ferrite matrix, a number density of $1 \times 10^{25} \mathrm{~m}^{-3}$ and a detection efficiency of $60 \%$ is shown in Fig. 3. Each precipitate contains $\sim 350$ atoms of which $\sim 190 \mathrm{Cu}$ atoms are detected. A cylinder of analysis with a $1 \mathrm{~nm}$-diameter probe aperture was placed through this volume.

The resulting composition profile, based on a typical sample size of 7844 atoms, intersects 10 precipitates and is shown in Fig. 4. Under these experimental parameters, it is evident that the true copper concentration is measured in some of these precipitates encountered whereas, a reduced concentration amplitude is measured in the others. In this typical simulation, 4 out of 10 precipitates are correctly sampled. However, it should be noted that in additional simulations of the same experimental parameters, there were significant variations in the total number of precipitates intersected. As expected, the maximum concentration observed for a precipitate is not necessarily the best estimate of its true composition since values greater than the average value are possible due to counting statistics.

\section{GEOMETRICAL MODEL}

In an atom probe analysis of spherical precipitates, the cylinder of analysis intersects precipitates as shown schematically in Fig. 5. The relative contribution from the precipitate is calculated by adding the two areas $A_{1}$ and $A_{2}$ formed by the chord joining the intersections of the two circles [2]. The maximum contribution from a precipitate of radius $r_{p}$ is therefore

$$
\mathrm{C}=\frac{\mathrm{A}_{1}+\mathrm{A}_{2}}{\pi \mathrm{rap}^{2}}
$$

where the areas $A_{1}=r_{a p}^{2}\left(\theta_{1}-\sin \left(\theta_{1}\right) \cos \left(\theta_{1}\right)\right)$ and $A_{2}=r_{p}^{2}\left(\theta_{2}-\sin \left(\theta_{2}\right) \cos \left(\theta_{2}\right)\right)$ and $\theta_{1}$ and $\theta_{2}$ are the angles subtended by the chord joining the intersections of the circles at the center of the probe aperture projection and axis of the particle, respectively, and are given by

$$
\cos \theta_{1}=\frac{x^{2}+r_{a p}{ }^{2}-r_{p}{ }^{2}}{2 x r_{a p}} \text { and } \cos \theta_{2}=\frac{x^{2}+r_{p}{ }^{2}-r_{a p}^{2}}{2 x r_{a p}} \text {. }
$$

where $\mathrm{x}$ is the distance from the centre of the precipitate to the axis of the cylinder of analysis. The probability of measuring the correct composition of a 2-nm-radius spherical precipitate and for four different effective radii of the probe aperture is shown in Fig. 6. Under the same experimental parameters as used in the simulation (i.e., 2-nm-diameter precipitate and 1-nm-diameter probe aperture), the geometrical model predicts that the correct concentration should be measured in $-35 \%$ of the precipitates encountered in good agreement with the atom probe simulator results $(40 \%)$. The probability of measuring the correct composition of a precipitate as a function of precipitate size can be extracted from these data and is summarized in Fig. 7. As the size of the precipitate increases with respect to the size of the probe aperture, the number of precipitates that are cleanly sampled also increases but at a relatively slow rate. This simple geometrical model indicates that even when a relatively large precipitate is sampled by a small diameter probe aperture, there will be a significant number of precipitates in which the true composition is not measured. A general rule would indicate that the probe aperture should be less than $1 / 3$ of the diameter of the precipitates in order that the correct composition is measured in a significant proportion (e.g., $\sim 40 \%$ ) of the precipitates. It is important that a statistically significant number of precipitates are sampled. In cases where the precipitate size is comparable to the effective size of the probe aperture, a better approach to determine the true composition of a precipitate may be to fit the experimental data to the data from an appropriate geometrical model.

It is apparent that the size of the precipitate may be an important factor in determining the composition of a precipitate. One method of determining the size is to use the persistence size method (i.e., to count the number of crystallographic planes of material that are field evaporated while the precipitate is visible in the field ion image). An alternative method is to estimate the size from the composition profile. Due to the spherical shape of the precipitate, the radius of the precipitate sampled at position $\mathrm{z}$ along the cylinder of analysis, $\mathrm{r}_{\mathrm{z}}$, will 

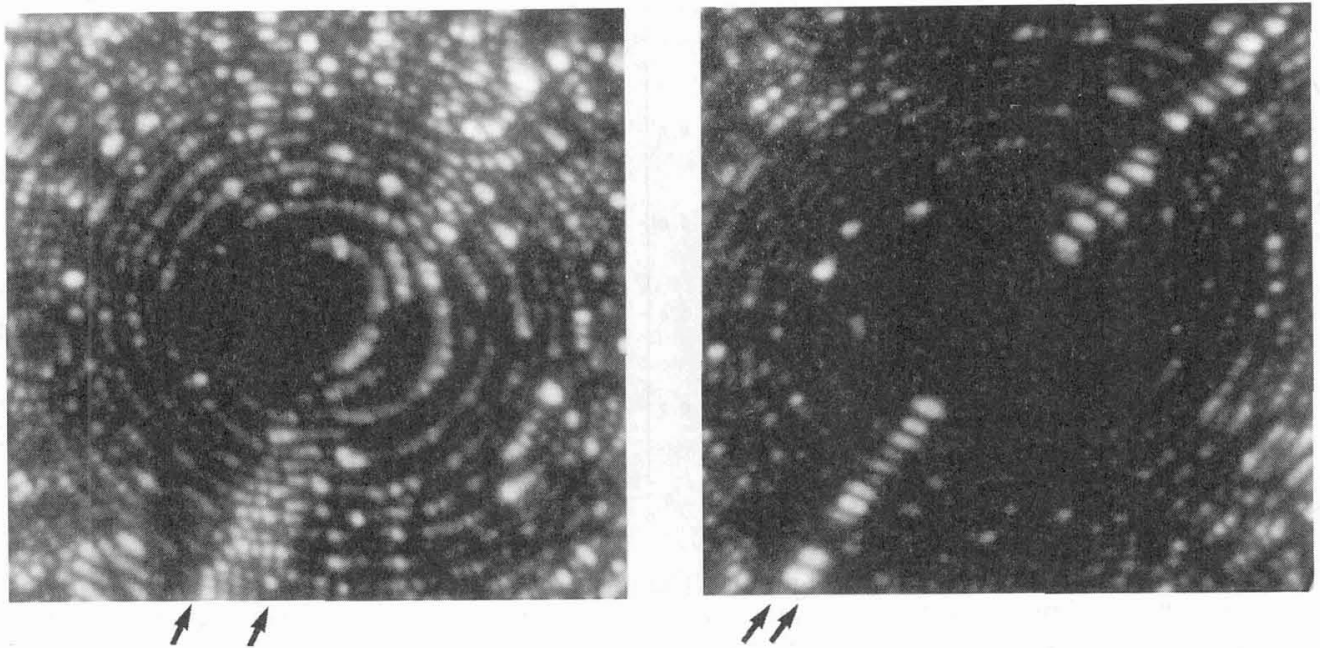

Figure 1. Field ion micrographs of thin platelets in an model nickel-based superalloy containing 10 at. \% Be.
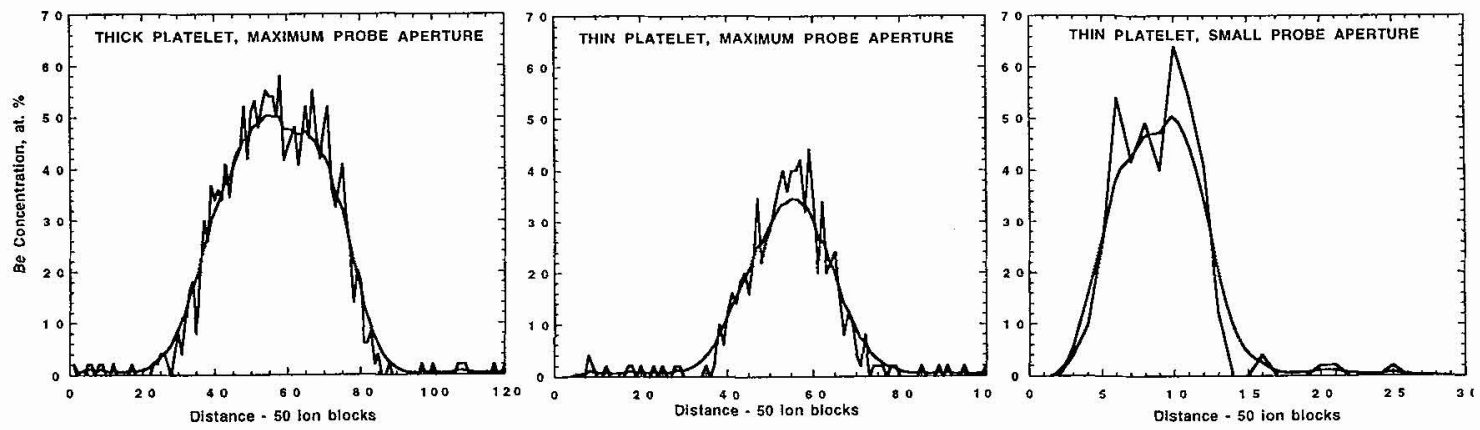

Figure 2. Series of composition profiles across the thin NiBe platelets in the $\gamma^{\prime}$ phase of a model nickelbased superalloy shown in Fig. 1.
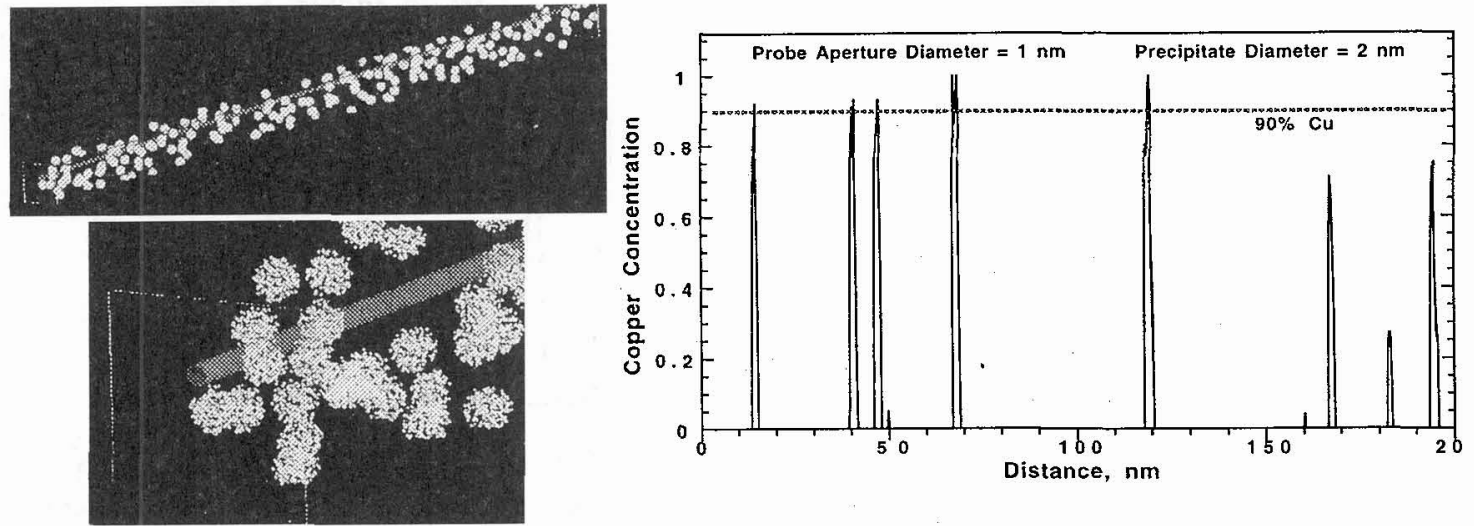

Figure 3. Simulation of randomly-distributed non-impinging 2- Figure 4. Composition profile through the nm-diameter coherent bec precipitates with a composition of Fe- data shown in Fig. 3. The probe aperture $90 \% \mathrm{Cu}, \mathrm{N}_{\mathrm{V}}=1 \times 10^{25} \mathrm{~m}^{-3}$ and a detection efficiency of $60 \%$. was $1 \mathrm{~nm}$ in diameter. 


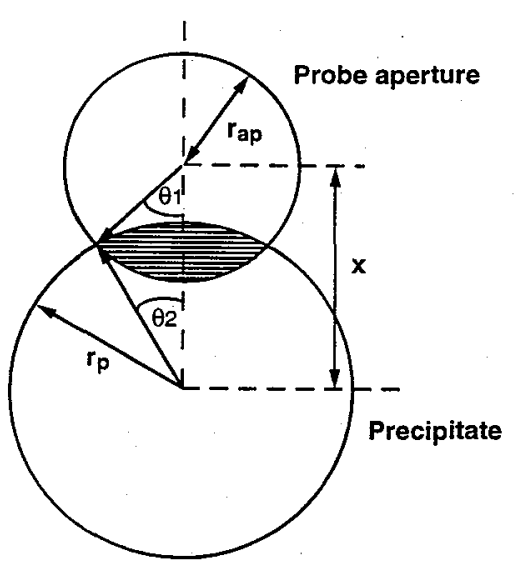

Figure 5. The geometrical model of the intersection of a spherical precipitate with the cylinder of analysis.

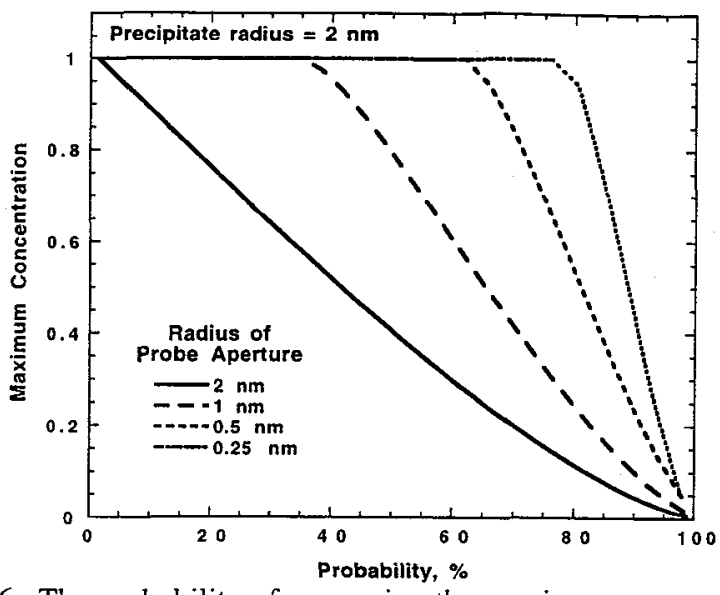

Figure 6 . The probability of measuring the maximum composition in an atom probe random area analysis for a $2-\mathrm{nm}$ radius precipitate and four different probe apertures.

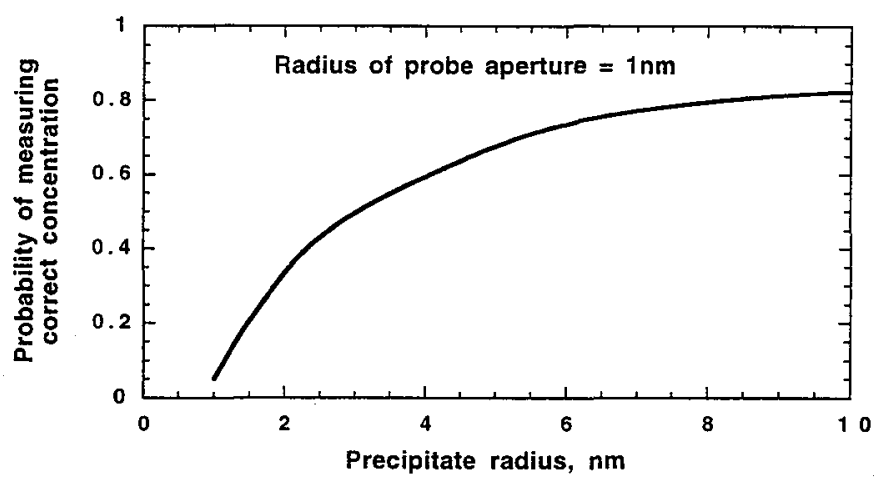

Figure 7. The probability of measuring the true composition of a precipitate for an effective probe radius of $1 \mathrm{~nm}$.

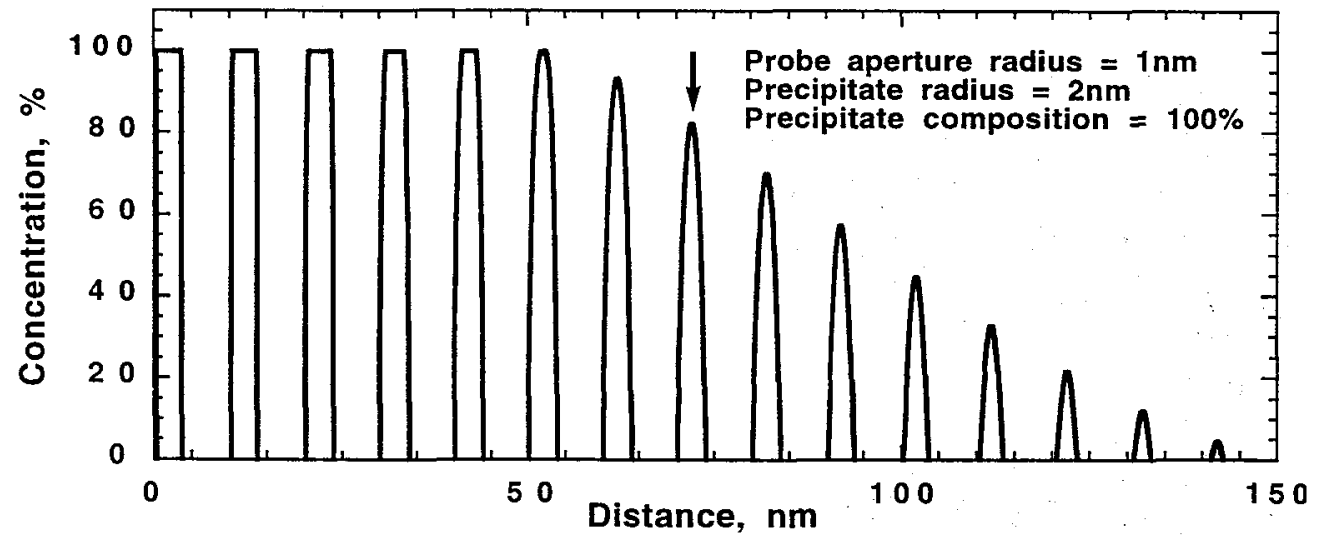

Figure 8. A series of composition profiles as the axis of the cylinder of analysis is moved from the center of a 2-nm-radius precipitate showing the difference in size and concentration measured. The first six precipitates are fully sampled. 
be less than the radius of the precipitate and is given by $r_{z}=\sqrt{r_{p}^{2}-z^{2}} . \quad$ A series of composition profiles with a $2 \mathrm{~nm}$ diameter probe aperture through a 4-nm-diameter precipitate with a solute content of $100 \%$ is shown in Fig. 8. The maximum diameters measured are shown in Fig. 9. The maximum extent of a precipitate measured in a composition profile is related to the diameter of the precipitate. The correct size is measured in a higher percentage of precipitates than the correct composition as shown by the arrows in Figs. 8 and 9 .

It is interesting to invesitgate whether it would be possible to distinguish between a fully sampled precipitate and a partially sampled precipitate with different compositions based on the shapes of their measured concentration profiles. The composition profiles for two different partially sampled precipitates containing $100 \%$ solute and two precipitates containing $50 \%$ solute in which the correct concentration was measured are shown in Fig. 10. In theory, it should be possible to distinguish between these cases since the shapes of the measured concentration profiles are slightly different. However, it unlikely to be achievable in practice since insufficient samples are available due to the small number of atoms.

The size of the precipitate is also an important parameter in the determination of the number density of precipitates. The number density of precipitates, $N_{V}$, is given by $N_{V}=N_{L} / \pi r^{2}$, where $N_{L}$ is the number of precipitates intersected per unit length and $\mathrm{r}$ is the radius of the precipitate [3]. This equation evaluates to 1.6 ( \pm 1.0) $\times 10^{25} \mathrm{~m}^{-3}$ for the example shown in Fig. 3. This example also provides an estimate of the number of precipitates that would typically be encountered in an atom probe experiment. For example, if the number density of precipitates was reduced by a factor of 10 to $1 \times 10^{24} \mathrm{~m}^{-3}$ only 1 precipitate would be encountered on average in this cylinder of analysis and therefore, the amount of data required should be increased appropriately.

\section{ADDITIONAL FACTORS}

The models and the simulations have been applied to simplified cases. However, in atom probe experiments, many additional factors including preferential evaporation and retention, different atomic densities of the precipitate and matrix, concentration gradients in the precipitate and matrix, variations in precipitate size, local compositional inhomogenieties, field-induced surface segregation, etc. will complicate the estimation of the microstructural parameters. An example of increasing the matrix concentration to $10 \%$ solute in the simulation is shown in Fig. 11. It is difficult to distingush a partially intersected precipitate from the matrix solute.

Some of these factors may be reduced or eliminated by the use of different experimental parameters such as specimen temperature, pulse fraction and pulse repetition rate. Other factors, such as the different atomic densities, may be relatively easily taken into account if the data are known. The deconvoltion is also simplified if a constant probe aperture is used during the atom probe experiment.

\section{CONCLUSIONS}

These simulations and the geometrical model indicate that the diameter of the probe aperture should be less than approximately $50 \%$ the diameter of the precipitates in order that the correct composition is measured in a significant proportion of the precipitates. The major limitations in the quantification of the composition and size of ultrafine precipitates is the small number of solute atoms available for analysis in each precipitate and the small number of precipitates intersected in an atom probe analysis. It is evident that care should be exercised in the interpretation of atom probe data from materials with low number densities of ultrafine precipitates since the small number of precipitates encountered in a typical atom probe experiment may not provide a true representation of the microstructure.

\section{Acknowledgments}

The authors would like to thank the late Dr. M. G. Hetherington and Drs. J. M. Hyde and A. Cerezo of Oxford University for many fruitful discussions. This research was sponsored by the Division of Materials Sciences, U. S. Department of Energy, under contract DE-AC05-96OR22464 with Lockheed Martin Energy Research Corp. This research was conducted utilizing the Shared Research Equipment (SHaRE) User Program facilities at Oak Ridge National Laboratory.

\section{References}

[1] Miller M. K. and Hyde J. M., Proc. Eurem'96, August 1996, Dublin, Ireland.

[2] Miller M. K., Cerezo A, Hetherington M. G. and Smith G. D. W., Atom Probe Field Ion Microscopy, (Oxford University Press, Oxford, England, 1996).

[3] Underwood E. E., in Quantitive Microscopy, eds. DeHoff R.T. and Rhines F.N., (McGraw-Hill, New York, 1968) pp.149-200. 


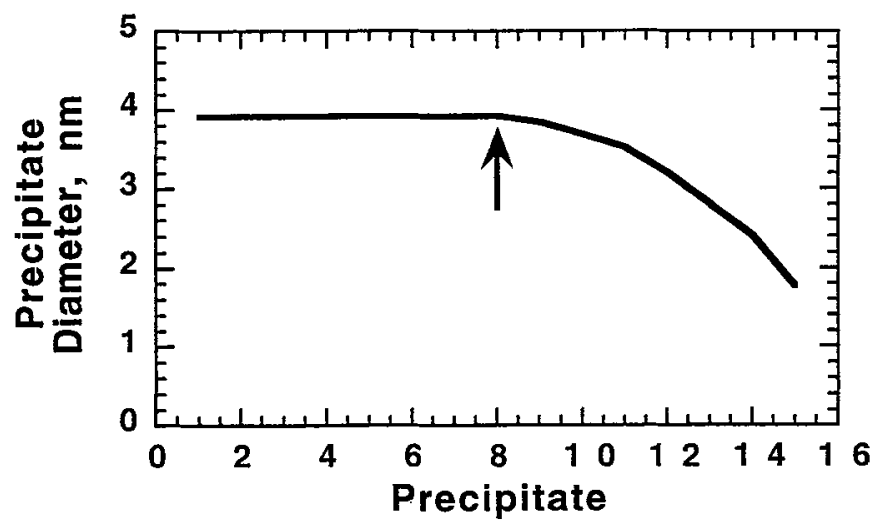

Figure 9. The size estimates of the 15 precipitates shown in Fig. 9. The size was taken as the maximum extent exhibited in the composition profile.

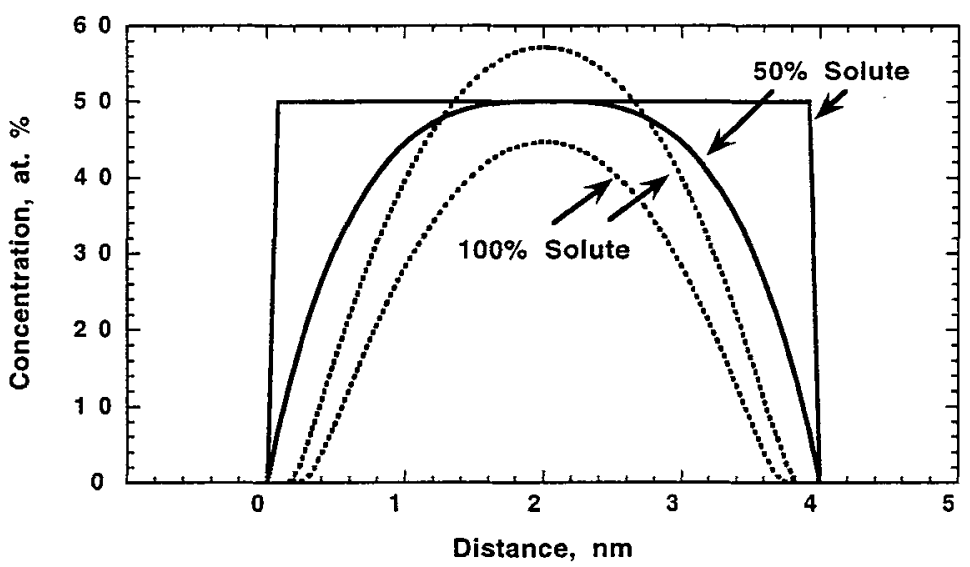

Figure 10. Comparison of the shape of the concentration profiles for four 4-nm-diameter precipitates. Solid lines represent precipitates containing 50\% solute in which the correct concentration is measured; dashed lines represent partially intersected precipitates containing $100 \%$ solute. The fully-sampled precipitates are always wider.

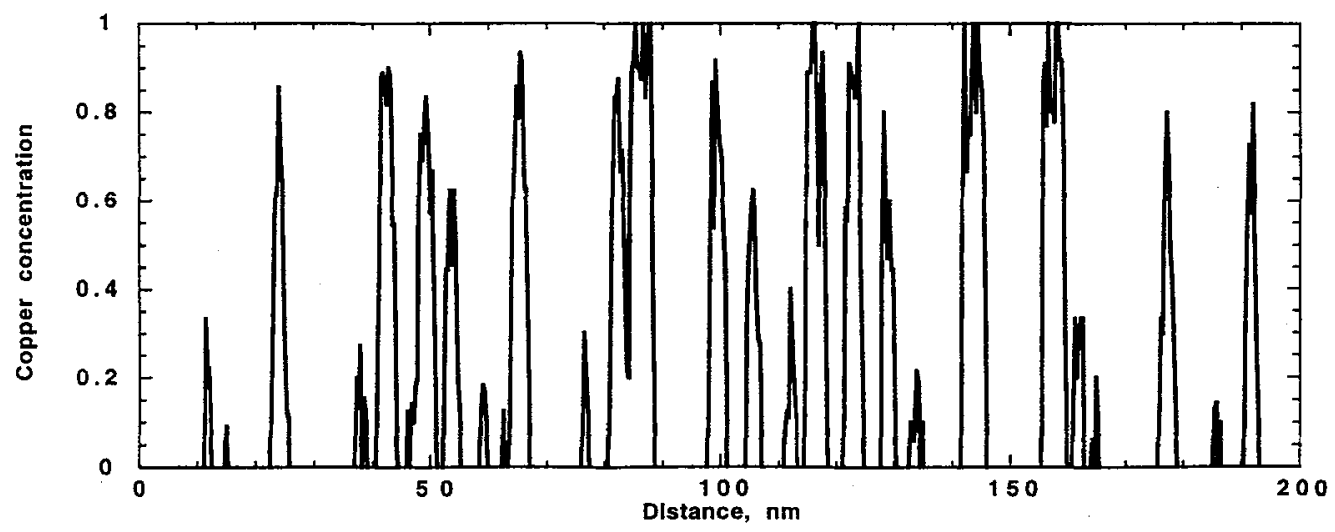

Figure 11. Simulated composition through precipitates containing $90 \%$ solute in a matrix containing $10 \%$ solute. 